\title{
Telomere length is positively associated with the expression of IL-6 and MIP-1 $\alpha$ in bone marrow mesenchymal stem cells of multiple myeloma
}

\author{
SHENGLI LI ${ }^{1-3^{*}}$, YANG JIANG ${ }^{1,3^{*}}$, AI LI $^{1,3}$, XIAOLI LIU ${ }^{1,3}$, XIANGLING XING ${ }^{1,3}$, YANAN GUO $^{1,3}$, \\ YAQI XU ${ }^{1,3}$, YUNLIANG HAO ${ }^{2}$ and CHENGYUN ZHENG ${ }^{1,3,4}$ \\ ${ }^{1}$ Department of Hematology, The Second Hospital, Institute of Biotherapy for Hematological Malignancies, \\ Shandong University, Jinan, Shandong 250033; ${ }^{2}$ Department of Hematology, Jining No. 1 People's Hospital, Jining, \\ Shandong 272100; ${ }^{3}$ Shandong University-Karolinska Institutet Collaboration Laboratory for Stem Cell Research, Jinan, \\ Shandong 250033; ${ }^{4}$ Shenzhen Research Institute of Shandong University, Shenzhen, Guangdong 518057, P.R. China
}

Received July 31, 2016; Accepted May 9, 2017

DOI: $10.3892 / \mathrm{mmr} .2017 .6885$

\begin{abstract}
Potential roles of mesenchymal stem cells (MSCs) in the pathogenesis of multiple myeloma (MM) are largely unknown. In the current study, the authors analyzed telomere length and the expressions of interleukin (IL)-6 and macrophage inflammatory protein (MIP)-1 $\alpha$ in MSCs derived from the bone marrow (BM) of MM patients and controls. The current results demonstrated that there was no significant difference in cell surface expression of CD73 and CD90, and the capacity to differentiate into bone tissue were identified between the BM MSCs derived from MM patients and controls. Interestingly, telomere length (TL) and mRNA expressions of IL-6 and MIP-1 $\alpha$ were significantly longer or higher in BM MSCs of MM than those of controls. Moreover, TL is positively associated with the expressions of IL-6 and MIP- $1 \alpha$ at the mRNA level in BM MSCs of MM. Additionally, IL-6 and MIP-1 $\alpha$ expression were significantly upregulated when MSCs from MM patients were cultured in the myeloma associated condition medium. The present study indicated that myeloma-associated elongation of TL of BM MSCs may be a key element contributing to the increased IL-6 and MIP-1 $\alpha$ expression, by which MSCs in the tumor microenvironment may facilitate MM and/or MM bone disease development.
\end{abstract}

Correspondence to: Professor Chengyun Zheng, Shenzhen Research Institute of Shandong University, 19 Gaoxin South Road, Virtual University Park, Nanshan, Shenzhen, Guangdong 518057, P.R. China

E-mail: zhengchengyun186@126.com

*Contributed equally

Key words: telomere length, multiple myeloma, bone marrow mesenchymal stem cells, interleukin-6, macrophage inflammatory protein-1 alpha

\section{Introduction}

Multiple myeloma (MM) is an incurable B cell malignancy characterized by the clonal expansion of plasma cells within the bone marrow (BM). It is well established that the transformation of myeloma is not only dependent on genetic and epigenetic aberrations, but also on the interaction between the malignant plasma cells and the BM microenvironment $(1,2)$. The BM microenvironment is composed of a noncellular compartment including the extracellular matrix and the liquid milieu, and cellular compartments of mesenchymal origin (3). Myeloma cells modify this microenvironment through their production of regulatory factors and direct cell-to-cell contact, to create a milieu that promotes myeloma cell survival (4-6) and augments osteoclast $(\mathrm{OC})$ recruitment and $\mathrm{OC}$ mediated bone loss $(7,8)$.

As an essential cell type in the BM microenvironment, bone marrow mesenchymal stem cell (MSCs) is a type of adult stem cell endowed with self-renew and differentiation capacities (9). Abnormalities of MSCs derived from MM patients (MM-MSCs) have been described in previous studies (10-12). Compared with MSCs isolated from healthy donors, increased expressions of cytokines and extracellular matrix were detected in MM-MSCs $(11,13,14)$. Additionally, decreased osteogenic potentiality and reduced efficiency of suppression of T-cell proliferation were also described (15-17). As well, MM-MSCs exhibited an abnormal upregulation of microRNA-135b and promoted MM tumor growth $(18,19)$. Moreover, MM-MSCs demonstrated non-recurrent genomic imbalances and hypomethylation of some genomic region resulted in upregulated several microRNAs, which are involved in regulation of senescence status and cell cycle characteristics of MM-MSCs $(12,20)$.

Telomeres are composed of repetitive DNA sequences and associated proteins at the end of the chromosomes. They serve a dominant role in protecting the end of DNA from fusion, recombination and degradation (21). Telomeric DNA shortens itself with each cell division due to the end-replication limitation of chromosomes (22). Correlation is reported between telomere 
length and replicative capacity of human fibroblasts (23). However, like most normal somatic cells, telomerase activity has not been detected in human MSCs (24). Telomeres of MSCs shortens its length concomitantly with the increased age or cell division $(25,26)$. Moreover, dysfunction of telomeres impairs function of MSCs and alters their gene expressions $(26,27)$.

In the current study, the authors compared the telomere length (TL) in bone marrow MSCs from MM patients and age-matched controls. The results demonstrated that the TL in MM-MSCs was longer than that in controls, and MM-MSCs secreted higher levels of interleukin (IL)-6 and macrophage inflammatory protein (MIP)- $1 \alpha$ than the controls. Moreover, the results indicated that the telomere length of MM-MSCs was closely correlated with the expressions of IL-6 or MIP-1 $\alpha$.

\section{Materials and methods}

Cell culture. The human myeloma cells RPMI-8226 were cultured in RPMI-1640 medium (Hyclone; GE Healthcare Life Sciences, Logan, UT, USA) containing $10 \%$ fetal bovine serum with the addition of $2 \mathrm{mM}$ L-glutamine (both from Gibco; Thermo Fisher Scientific, Inc., Waltham, MA, USA) and antibiotics $(100 \mathrm{U} / \mathrm{ml}$ penicillin and $0.1 \mathrm{mg} / \mathrm{ml}$ streptomycin) in a humid atmosphere at $37^{\circ} \mathrm{C}$ and $5 \% \mathrm{CO}_{2}$. After cell culture for $24 \mathrm{~h}$, the conditioned medium of RPMI-8226 was collected and stored in $-20^{\circ} \mathrm{C}$.

Isolation and expansion of MSCs from bone marrows. Bone marrow (BM) samples were obtained from patients with MM or from controls (patients without a tumor, infection or autoimmune diseases; Table I). The current study was approved by the Ethics Committee of the Second Hospital of Shandong University (Jinan, China) and informed consent was obtained from all patients. Mononuclear cells (MNCs) were obtained from BM samples by density gradient separetion using Ficoll density gradient centrifugation. Finally, MNCs were cultured in Dulbecco's modified Eagle's medium (Hyclone; GE Healthcare Life Sciences) supplemented with $10 \%$ defined fetal bovine serum (Shanghai ExCell Biology, Inc., Shanghai, China), growth factors (R\&D Systems, Inc., Minneapolis, MN, USA). At 72 or $96 \mathrm{~h}$ incubation, non-adherent cells were carefully removed, whereas the MSCs attached to the cell culture plate were defined as passage 0 (P0) MSCs. When the MSCs reached approximately $80 \%$ confluence, the cells were passaged (P1) by digestion using $0.05 \%$ trypsin-EDTA (Thermo Fisher Scientific, Inc.) The MSCs were collected near confluency at $\mathrm{P} 4$ for subsequent experiments.

Osteoblastic differentiation of MSCs. An osteocyte differentiation assay was performed according to the instructions provided in the StemPro ${ }^{\circledR}$ Osteogenesis Differentiation kit (cat no. A1007201; Thermo Fisher Scientific, Inc.). Briefly, MSCs were seeded at $1 \times 10^{4} / \mathrm{cm}^{2}$ in 6 -well culture plates in osteoblast differentiation culture media for 21 days. Medium was changed 2-3 times a week. The osteocytes were stained with Alizarin red (cat no. A5533; Sigma-Aldrich, Merck KGaA, Darmstadt, Germany).

Immunophenotyping of bone marrow MSCs. The bone marrow MSCs were labeled with phycoerythrin-conjugated anti-CD73 (cat no. 550257), fluorescein isothiocyanate (FITC)-conjugated anti-CD90 (cat no. 555595), FITC-conjugated anti-CD105 (cat no. 561443) (all from BD Biosciences, Franklin Lakes, NJ, USA) and human leukocyte antigen G (HLA-G; cat no. 12-9957-73; eBioscience, Inc., San Diego, CA, USA). The fluorescence of the above MSC surface markers was detected and analyzed using flow cytometry with a BD FACSAria ${ }^{\mathrm{TM}}$ II system (BD Biosciences). Data were analyzed using the FlowJo software version 7.6 (FlowJo, LLC, Ashland, Oregon, USA).

Quantitative polymerase chain reaction $(q P C R)$ and Flow-FISH for MSC telomere length assay. For qPCR detection, genomic DNA was isolated using QiAamp DNA Blood Mini kit (Qiagen GmbH, Hilden, Germany). Telomere length was determined by qPCR as described previously (28). The primer sequences for human telomere (Tel) and for $\beta$-globin (HBG) are listed in Table II. The relative telomere length was decided as the percentage of that in a MM-MSC. Flow-FISH of MSCs was performed as described previously (29).

Cell cycle analysis. Cell cycle analysis was performed using the Cell Cycle and Apoptosis Analysis kit (cat no. C1052-1; Beyotime Institute of Biotechnology, Haimen, China) and flow cytometry with a BD FACSAria ${ }^{\mathrm{TM}}$ II system (BD Biosciences) was conducted following the manufacturer's instructions. The percentage of each phase in the cell cycle was calculated using cell cycle analysis software Modfit version LT 3.1 (Verity Software House, Topsham, ME, USA).

Reverse transcription-quantitative polymerase chain reaction (RT-qPCR). Total RNA was extracted using TRIzol reagent (Thermo Fisher Scientific, Inc.) following the manufacturer's instruction. cDNA was synthesized using the M-MLV enzyme (Thermo Fisher Scientific, Inc.) and an oligo-dT primer according to the protocol. qPCR was performed using SYBR-Green kit (Applied Biosystems; Thermo Fisher Scientific, Inc.) on an ABI 7700 detector (Applied Biosystems; Thermo Fisher Scientific, Inc.). All sequences of primers are listed in Table II. Thermocycling conditions were as follows: At $50^{\circ} \mathrm{C}$ for $2 \mathrm{~min}$, at $95^{\circ} \mathrm{C}$ for $10 \mathrm{~min}$, followed by 45 cycles at $95^{\circ} \mathrm{C}$ for $15 \mathrm{sec}$ and at $60^{\circ} \mathrm{C}$ for $1 \mathrm{~min}$. The expression level of each gene was calculated according to the comparative $\mathrm{Cq}$ method and normalized to GAPDH expression (30).

Statistical analysis. The Student's t-test, paired t-test or Fisher's exact test were used to compare differences between groups. Pearson's correlation was applied to analysis for relationships between variables. All the results were presented using GraphPad Prism software (version, 5; GraphPad Software, Inc., La Jolla, CA, USA). The data are shown as mean \pm standard error of the mean $\mathrm{P}<0.05$ was considered to indicate a statistically significant difference.

\section{Results}

The phenotype, growth characteristics and osteoblastic differentiation of MSCs. As presented in Fig. 1, MSCs were cultured and analyzed of both MM patients (Fig. 1A) and the control group (Fig. 1B). The phenotypes were analyzed using flow cytometry. As presented in Table III, MSCs from 
Table I. Clinical characteristics of patients with multiple myeloma $(n=12)$.

\begin{tabular}{|c|c|c|c|c|c|c|}
\hline Sample & Gender & Age (years) & Disease stage $^{a}$ & $\beta 2-\mathrm{MG}(\mathrm{mg} / \mathrm{dl})$ & BM plasma cells $(\%)$ & Bone lesions \\
\hline 1 & $\mathrm{~F}$ & 68 & Early & 3.31 & 58 & $\mathrm{~N}$ \\
\hline 2 & M & 65 & Early & 3.36 & 51 & $\mathrm{~N}$ \\
\hline 3 & $\mathrm{~F}$ & 69 & Late & 11.5 & 67 & $\mathrm{Y}$ \\
\hline 4 & $\mathrm{~F}$ & 63 & Early & 3.74 & 51 & $\mathrm{Y}$ \\
\hline 5 & M & 65 & Late & 12.4 & 65 & $\mathrm{Y}$ \\
\hline 6 & M & 74 & Late & 6.72 & 48 & $\mathrm{~N}$ \\
\hline 7 & $\mathrm{M}$ & 58 & Late & 7.79 & 32 & $\mathrm{Y}$ \\
\hline 8 & M & 57 & Late & 40.5 & 40 & $\mathrm{Y}$ \\
\hline 9 & M & 59 & Late & 7.89 & 4 & $\mathrm{Y}$ \\
\hline 10 & M & 70 & Late & 9 & 43 & $\mathrm{Y}$ \\
\hline 11 & M & 58 & Late & 20.8 & 20 & $\mathrm{~N}$ \\
\hline 12 & $\mathrm{~F}$ & 59 & Early & 4.25 & 32 & $\mathrm{Y}$ \\
\hline
\end{tabular}

a'Early, I and II; late, III. ISS staging system was used. N, no; Y, yes; M, male; F, female; $\beta 2-\mathrm{MG}, \beta 2$-microglobulin; BM, bone marrow.

Table II. The primer sequences for quantitative polymerase chain reaction and telomere length assessment.

\begin{tabular}{|c|c|c|}
\hline & Gene & Sequence $\left(5^{\prime}-3{ }^{\prime}\right)$ \\
\hline \multirow[t]{2}{*}{1} & IL-6 forward & GAGGCACTGGCAGAAAACAACC \\
\hline & IL-6 reverse & ССТСАААСТССААААGACCAGTGATG \\
\hline \multirow[t]{2}{*}{2} & MIP-1 $\alpha$ forward & GAATCATGCAGGTCTCCACTG \\
\hline & MIP- $1 \alpha$ reverse & CTCTAGGTCGCTGACATATTTC \\
\hline \multirow[t]{2}{*}{3} & IDO forward & TGGGGCAAAGGTCATGGAG \\
\hline & IDO reverse & TTTCTTGGAGAGTTGGCAGTAAG \\
\hline \multirow[t]{2}{*}{4} & hTERT forward & CGGAAGAGTGTCTGGAGCAA \\
\hline & hTERT reverse & GGATGA AGCGGAGTCTGGA \\
\hline \multirow[t]{2}{*}{5} & GAPDH forward & GCACCGTCAAGGCTGAGAAC \\
\hline & GAPDH reverse & TGGTGAAGACGCCAGTGGA \\
\hline \multirow[t]{2}{*}{6} & Tellb & CGGTTTGTTTGGGTTTGGGT-TTGGGTTTGGGTTTGGGTT \\
\hline & Tel $2 b$ & GGCTTGCCTTACCCTTACCCTTACCC-TTACCCTTACCCT \\
\hline \multirow[t]{2}{*}{7} & HBG3 & TGTGCTGGCCCATCACTTTG \\
\hline & HBG4 & ACCAGCCACCACTTTCTGATAGG \\
\hline
\end{tabular}

hTERT, human telomerase reverse transcriptase; IL, interleukin; MIP-1 $\alpha$, macrophage inflammatory protein-1 $\alpha$.

both MM patients and controls were positive for CD73, CD90 and CD105, but negative for HLA-G. There were no significant differences between MM patients (6.08 \pm 0.69 days) and controls (8.62 \pm 1.33 days) on the days for obtaining $80 \%$ confluent MSCs cultures. The osteoblastic differentiation of MSCs was examined using Alizarin red staining. As indicated in Fig. 2, MSCs from three age-matched pairs of MM patients (Fig. 2A) and controls (Fig. 2B) displayed deposition of matrix mineralization.

The TL and cell cycle of MSCs from MM patients and control group. The TL of the MSCs from $12 \mathrm{MM}$ patients and 8 controls were compared by qPCR. As reported in Fig. 3A, the TL of MSCs from MM patients was significantly longer
$(0.98 \pm 0.11)$ than the controls $(0.65 \pm 0.07 ; \mathrm{P}=0.03)$. The authors further assessed the TL in MSCs using Flow-FISH. Similarly, the TL of MSCs was longer in the MM patients $(10,860 \pm 807)$ than in the controls $(5,227 \pm 1,143 ; \mathrm{P}=0.015$; Fig. $3 \mathrm{~B})$. Cell cycle characteristics of MM-MSCs were determined using flow cytometry. As presented in Fig. 3C, the percentage of MM-MSCs in G0/G1 was increased compared with controls $(77.33 \pm 1.83$ vs. $61.87 \pm 2.51 ; \mathrm{P}<0.01)$, whereas the MM-MSCs in the $\mathrm{S}$ phase and $\mathrm{G} 2 / \mathrm{M}$ phase fell to $14.36 \pm 1.36 \%$ (vs. $26.98 \pm 3.22 ; \mathrm{P}<0.01)$ and $6.34 \pm 0.75 \%$ (vs. 10.95 $\pm 1.14 ; \mathrm{P}<0.01$ ) respectively, when compared to those of controls.

The human telomerase catalytic subunit gene hTERT, which is the putative human telomerase catalytic subunit gene serving as an indicator of telomerase activity (31), were also 
A

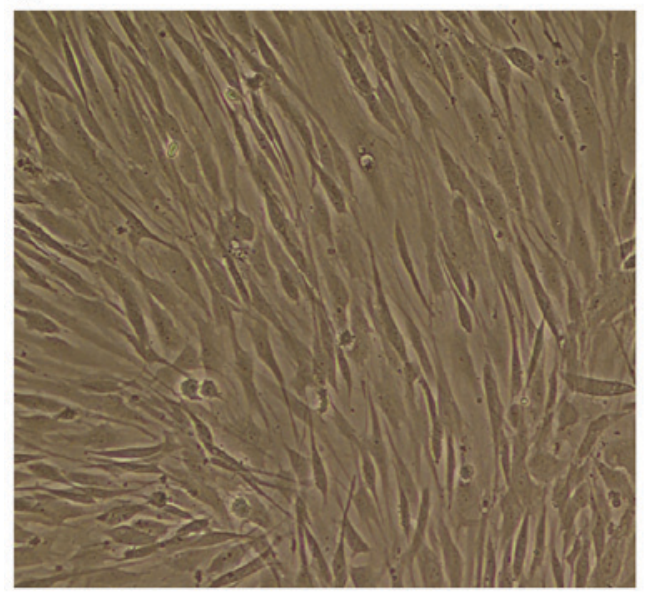

B

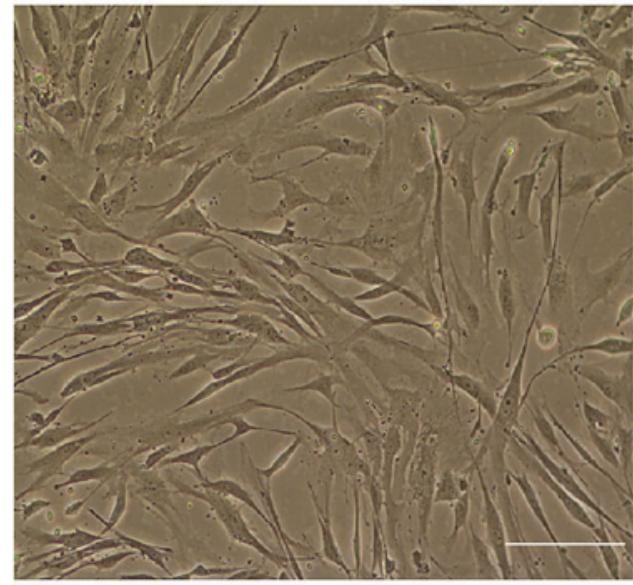

Figure 1. Bone marrow mesenchymal stem cells were cultured and analyzed at passage 4 of (A) patients with multiple myeloma and (B) the control group (magnification, x200; scale bar, $50 \mu \mathrm{m}$ ).

A

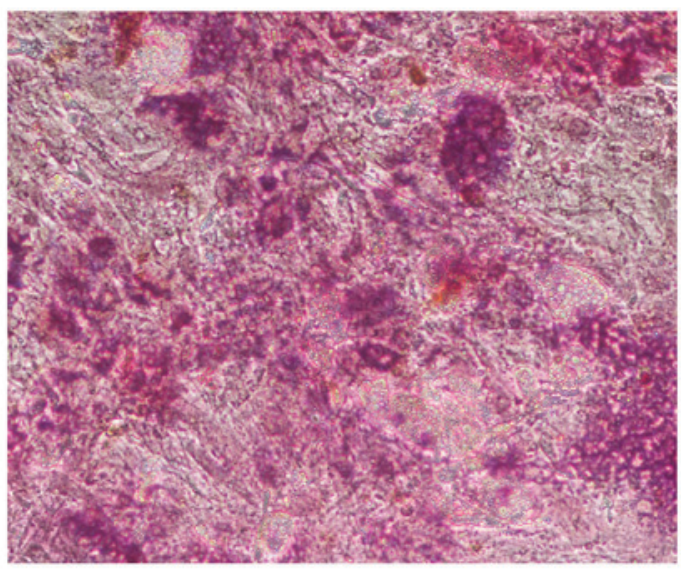

B

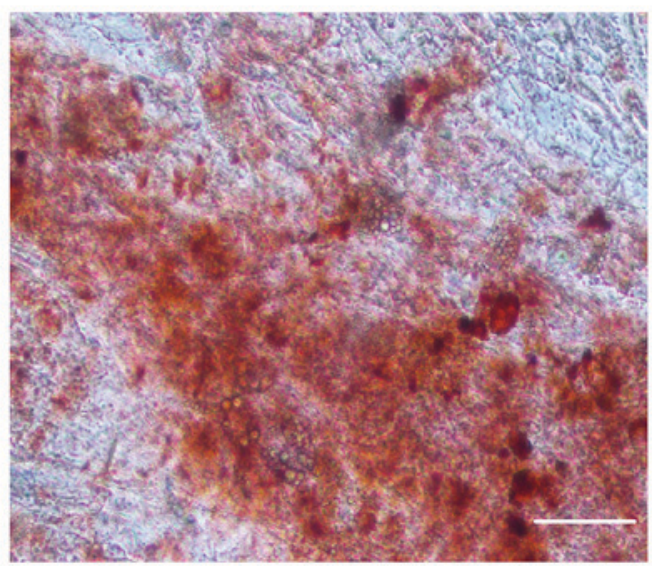

Figure 2. Osteoblastic differentiation detection of mesenchymal stem cells using Alizarin red staining of (A) patients with multiple myeloma and (B) the control group following 21 days of differentiation (magnification, x100; scale bar, $100 \mu \mathrm{m}$ ).

Table III. Flow cytometry analysis of mesenchymal stem cells from control and MM patients.

\begin{tabular}{lccc}
\hline $\begin{array}{l}\text { Antigen } \\
\text { Expression }\end{array}$ & $\begin{array}{c}\text { MM patients } \\
(\text { MEAN } \pm \text { SEM) }\end{array}$ & $\begin{array}{c}\text { Control group } \\
(\text { MEAN } \pm \text { SEM) }\end{array}$ & P-value $^{\mathrm{a}}$ \\
\hline CD73 (\%) & $90.9 \pm 0.03$ & $89.5 \pm 0.03$ & 0.73 \\
CD90 (\%) & $75.4 \pm 0.06$ & $83.4 \pm 0.05$ & 0.39 \\
CD105 (\%) & $62.6 \pm 10.4$ & $59 \pm 8$ & 0.81 \\
HLA-G $(\%)$ & $1.1 \pm 4.4$ & $0.9 \pm 0.5$ & 0.77 \\
\hline
\end{tabular}

${ }^{a}$ Comparison between patient group and control. CD, cluster of differentiation; HLA-G, human leukocyte antigen G; MM, multiple myeloma; SEM, standard error of the mean.

measured using RT-qPCR method in normal and MM-MSCs. However, hTERT expression in those cells was not detected (data not shown). These results indicated that both normal and MM-MSCs lacked telomerase activity.
The expressions of IL-6, indoleamine 2,3-dioxygenase (IDO) and MIP-1 $\alpha$ in MSCs from MM patients. The mRNA expressions of IL-6 and IDO in MSCs from MM patients and controls were investigated using RT-qPCR. The expression of IL-6 was significantly increased in MM-MSCs $(\mathrm{P}<0.05)$, while the expression of IDO decreased obviously compared with MSCs in control group ( $\mathrm{P}<0.05$; Fig. 3D). Moreover, the expression rate of MIP-1 $\alpha$ from MM-MSCs was significantly higher than that from MSCs in control group (91.7 and 37.5\% respectively, $\mathrm{P}<0.05)$.

The correlations between TL and IL-6, MIP-1 $\alpha$ and IDO. As presented in Fig. 4, the TL of MM-MSCs was correlated with their expression of IL-6 (Fig. 4A) and MIP-1 $\alpha$ (Fig. 4B). However, no significant correlation between the telomere length and the expression of IDO in MM-MSCs was found.

The conditioned medium of RPMI 8226 induces changes of gene expression and cell cycle in MSCs. MSCs isolated from three MM patients were cultured in myeloma condition culture medium (MCCM) (a mixture of myeloma cell 

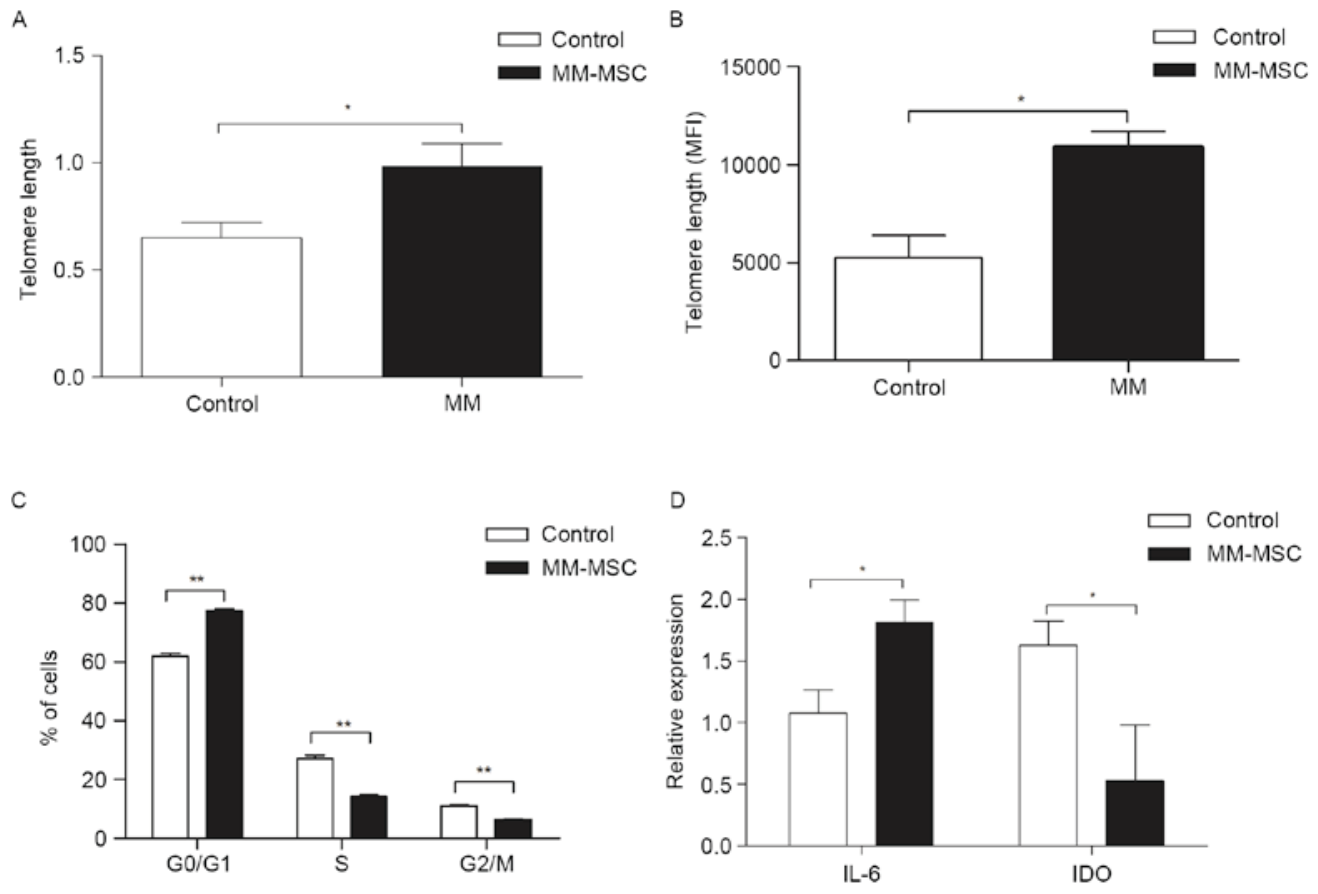

Figure 3. (A) Comparison of telomere length of MSCs using quantitative polymerase chain reaction between $12 \mathrm{MM}$ patients and 8 controls. (B) Comparison of telomere length in MSCs using Flow-fluorescent in situ hybridization between MM patients and controls $(\mathrm{n}=3)$. (C) Cell cycle analysis using flow cytometry in MM-MSCs ( $n=12)$ compared to the controls $(n=8)$. (D) The mRNA expressions of IL-6 and IDO in MSCs from MM patients and controls. Data are presented as the mean \pm standard error of the mean. ${ }^{*} \mathrm{P}<0.05,{ }^{* *} \mathrm{P}<0.01$ vs. control. IL, interleukin; IDO, indoleamine 2,3-dioxygenase; MSCs, mesenchymal stem cells; MM, multiple myeloma; MFI, median fluorescence intensity.
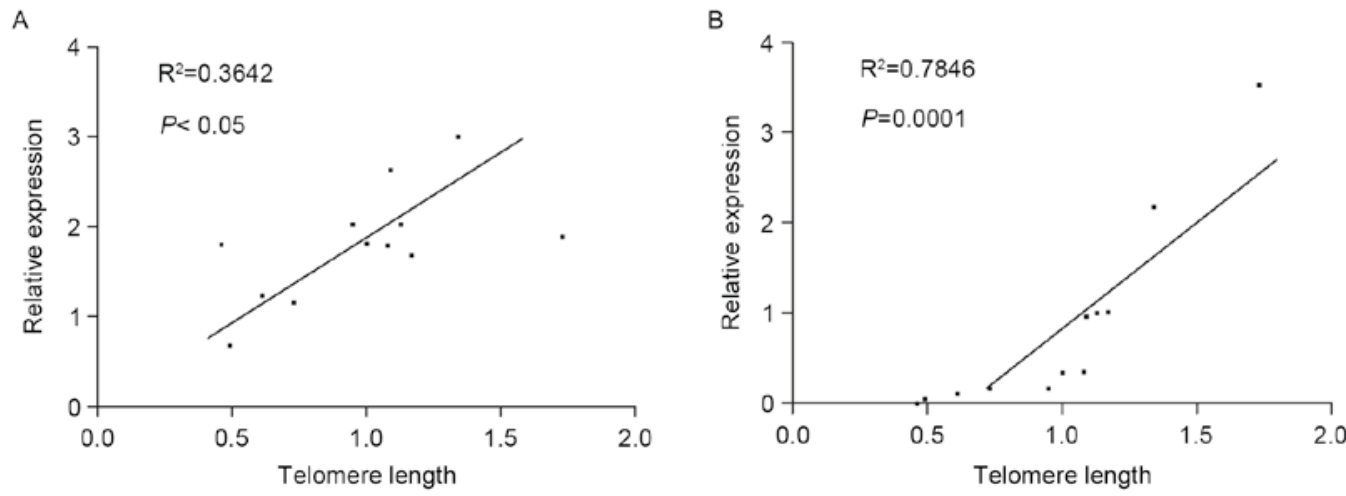

Figure 4. MM-MSCs telomere length was correlated with the expression of (A) IL-6 and (B) MIP-1 $\alpha$. The relative expression of IL-6 and MIP-1 $\alpha$ in MSCs from $12 \mathrm{MM}$ patients was plotted vs. telomere length. The telomere length of MM-MSCs correlates postitively with the expressions of (A) IL-6 and (B) MIP-1 $\alpha$. MM, multiple myeloma; MSCs, mesenchymal stem cells; IL, interleukin; IDO, indoleamine 2,3-dioxygenase; MIP-1 $\alpha$, macrophage inflammatory protein-1 $\alpha$.
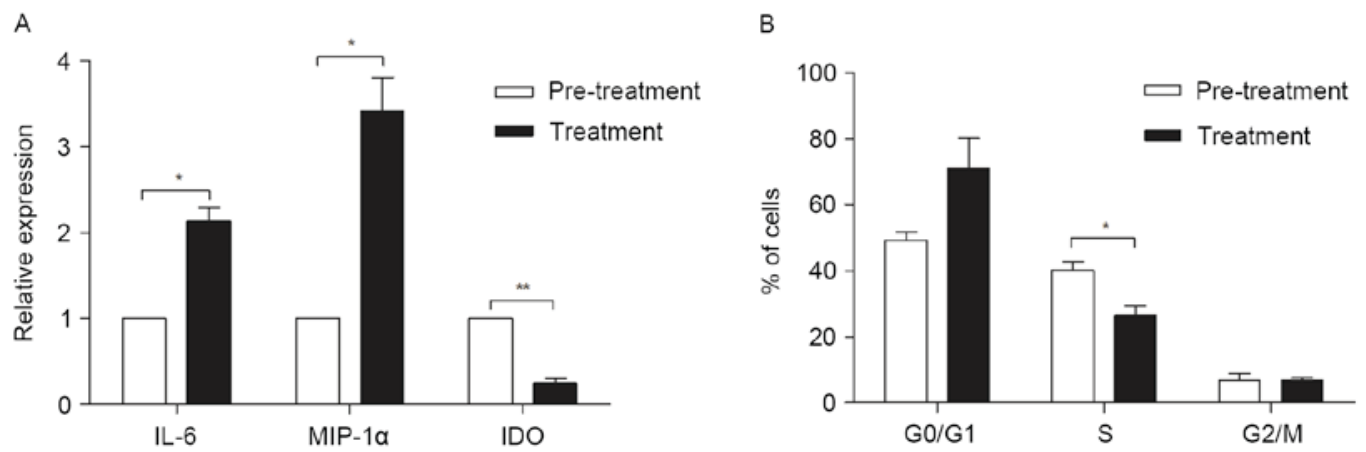

Figure 5. (A) mRNA expressions of IL-6, MIP-1 $\alpha$ and IDO before and after the culture with myeloma condition medium (a mixture of supernatant of myeloma cell line RPMI 8226 and MSCs complete medium). (B) Cell cycle analysis of MM-MSCs before and after the culture with mixed medium. Data are presented as the mean \pm standard error of the mean. ${ }^{*} \mathrm{P}<0.05$ as indicated. IL, interleukin; MIP-1 $\alpha$, macrophage inflammatory protein-1 $\alpha$; IDO, indoleamine 2,3 -dioxygenase; MM, multiple myeloma; MSCs, mesenchymal stem cells. 
line RPMI-8226 culture supernatant and MSCs complete medium) for $24 \mathrm{~h}$, then the expressions of IL-6, MIP-1 $\alpha$ and IDO were quantified using qPCR. When cultured in the MCCM, IL-6 $(\mathrm{P}<0.05)$ and MIP-1 $\alpha(\mathrm{P}<0.01)$ were significantly increased, while IDO was markedly downregulated $(\mathrm{P}<0.05)$, when compared with the MSCs cultured in regular MSC medium (Fig. 5A).

To explore whether MCCM affects the cell cycle of MM-MSCs, MSCs from three MM patients were cultured in MCCM. At $24 \mathrm{~h}$ culture, MSCs reported an increase of cells in $\mathrm{G}_{0} / \mathrm{G}_{1}$ phase and a decrease of the cells in $S$ phase compared to the controls $(\mathrm{P}<0.05$; Fig. 5B).

The changes of MSCs were not correlated with $\beta 2$ microglobulin and bone marrow plasma cells. No significant correlations between TL and serum $\beta 2$ microglobulin ( $\beta 2-\mathrm{MG})$ $(\mathrm{r}=-0.24 ; \mathrm{P}=0.45)$, and the percentage of plasma cell in $\mathrm{BM}$ $(\mathrm{r}=0.55 ; \mathrm{P}=0.07)$ was identified. Moreover, these data did not present a correlation of IL- 6 or MIP- $1 \alpha$ expression with serum $\beta 2-\mathrm{MG}$, as well as the percentage of plasma cells in BM (data not shown).

\section{Discussion}

The direct and indirect interactions between BM-MSCs and MM cells not only mediate MM cell growth and survival, but also lead to the intrinsic abnormalities observed in MM-MSCs, such as genomic imbalances and an overexpression of IL-6 (32). In the present study, the authors indicated that bone marrow MSCs from MM are not different from control group in terms of phenotypic markers (e.g., CD73 and CD90) and capacity to differentiate into osteogenic tissues. However, the TL and expressions of IL- 6 and MIP- $1 \alpha$ were significantly increased in MM BM-MSCs than the controls. Moreover, the correlation between TL and IL- 6 as well as MIP-1 $\alpha$ expression in MM-MSCs were displayed.

The current results demonstrated that TL of MM-MSCs was significantly longer than that of controls, indicating a tumor or myeloma associated mechanism contributing to maintenance or elongation of TL. It is known that TLs shorten accordingly when cells divide. The authors' cell cycle analysis demonstrated that the percentage of MSCs in the $G_{0} / G_{1}$ phase was significantly increased in MM than in the controls, suggesting that there are more MM-MSCs in stable or non-dividing phase, which may in part explain why MM-MSCs present longer TL than the controls. In line with a previous report (24), these data suggested that MSCs from both controls and MM patients lacked telomerase activity. To date, the underlying the mechanisms of TL maintenance in MSCs is largely unknown. In MM cells lines, IL-6 and IGF-1 was demonstrated to increase telomerase activity via AKT-mediated phosphorylation of hTERT without alteration of the expression of hTERT at the level of mRNA or protein (33), indicating that MSCs may also apply this mechanism to maintain their TL in the microenvironment riched in inflammatory cytokines, such as IL-6.

Moreover, reduced percent of MSCs in S phase during cell cycle and increased in $G_{0} / G_{1}$ phase were identified following conditional culture. This result is in contrast with a recent report displaying that co-culture with $\mathrm{MM}$ cells produced a marked increase in $\mathrm{S} / \mathrm{G}_{2} / \mathrm{M}$ phases of the cells (10). These differences may be due to divergences in the in vitro culture system applied or the myeloma condition medium, but not cell-cell direct contact in our system.

In the present study, the authors also compared MSC differentiation capacity between MM patients and controls, and did not identify markedly difference in differentiation into bone tissue between them. This result is controversial compared to the previous reports that indicate that the differentiation capacity of MSCs from MM patients is lower than those from the normal controls $(19,34)$. It may be speculated that the primary reason causing the different results may be due to different cell passages applied in our and other studies. Since MSCs were used from passage 4, the in vitro culture or expansion procedures may overcome subtle function abnormalities of MM-MSCs. This issue will be addressed in future studies.

Changes in the expression of cytokines and growth factors from MSCs serve a prominent role in the growth and survival of multiple myeloma (1). High expression of IL-6 from MM-MSCs was detected. MM-MSCs were expressed a significantly higher level of IL-6 following culturing with the supernatants from MM cultures. Our findings are consistent with those previous reports that MM-MSCs overproduced IL-6 (35) and MM cells stimulated IL-6 expression in MSCs (4).

It has been demonstrated that MIP- $1 \alpha$ contributes to the development of bone disease in MM via tumor survival promotion $(36)$, osteoblast differentiation $(7,8)$ and osteoblast functional inhibition (37). MIP-1 $\alpha$ is indicated to be primarily produced by myeloma cells (8). Interestingly, MIP-1 $\alpha$ was shown to also be expressed in MM-MSCs, and the expression of MIP-1 $\alpha$ in MM-MSCs was higher than that in MSCs from the control. Moreover, MIP-1 $\alpha$ expression in MM-MSCs was further increased under the stimulation of the supernatants isolated from MM cell line culture. Therefore, MIP-1 $\alpha$ produced by MM-MSCs may also participate in the development of MM.

Previous studies have illustrated that the proliferative potential of human hematopoietic cells (38) and human fibroblasts (23) correlates with their telomere lengths. Similarly, the correlation between growth of MSCs and telomere length has also been suggested (39). Moreover, dysfunction of telomere impairs the function of mesenchymal progenitor cells and affects the expressions of various cytokines (27). Consistently, our findings of positive correlation of IL-6 and MIP- $1 \alpha$ with telomere lengths in MM-MSCs highlight that telomere lengths affect cytokine expression in MM-MSCs, by which MM-MSCs facilitate MM development.

IDO is an important immunoregulatory molecule (40). Our results demonstrated that IDO was significantly lower in MM-MSCs than controls. Supernatants derived from MM cell line further downregulated IDO expression. The IDO expression may result in impaired immunomodulatory capacities of BM-MSCs in MM $(15,17)$. Undoubtedly, the significance of such decreased IDO expression in MM-MSCs needs to be investigated in the future studies.

In summary, in the present study, the authors find that MM-MSCs present longer telomere and higher IL-6 and MIP-1 $\alpha$ expression, and telomere lengths are positively 
correlated with IL-6 and MIP-1 $\alpha$ expression. These results indicated that MSCs in BM may be involved in pathothegenesis of $\mathrm{MM}$ and $\mathrm{MM}$ related bone diseases.

\section{Acknowledgements}

The present study was supported by the National Natural Science Foundation of China (grant nos. 81372545 and 81600176), the Fundamental Research Funds of Shandong University (grant no. 2014QY004-16 and 2015QY002-10), Youth Fund of Second Hospital of Shandong University (grant no. Y2013010029), the Natural Science Foundation of Shandong Province (grant no. ZR2016HB71) and the Shenzhen Strategic Emerging Industry Development Special Fund (grant no. JCYJ20150402105524048).

\section{References}

1. Bianchi G and Munshi NC: Pathogenesis beyond the cancer clone(s) in multiple myeloma. Blood 125: 3049-3058, 2015.

2. Hideshima T, Mitsiades C, Tonon G, Richardson PG and Anderson KC: Understanding multiple myeloma pathogenesis in the bone marrow to identify new therapeutic targets. Nat Rev Cancer 7: 585-598, 2007.

3. Manier S, Sacco A, Leleu X, Ghobrial IM and Roccaro AM: Bone marrow microenvironment in multiple myeloma progression. J Biomed Biotechnol 2012: 157496, 2012.

4. Dankbar B, Padró T, Leo R, Feldmann B, Kropff M, Mesters RM, Serve H, Berdel WE and Kienast J: Vascular endothelial growth factor and interleukin-6 in paracrine tumor-stromal cell interactions in multiple myeloma. Blood 95: 2630-2636, 2000.

5. Gupta D, Treon SP, Shima Y, Hideshima T, Podar K, Tai YT, Lin B, Lentzsch S, Davies FE, Chauhan D, et al: Adherence of multiple myeloma cells to bone marrow stromal cells upregulates vascular endothelial growth factor secretion: Therapeutic applications. Leukemia 15: 1950-1961, 2001.

6. Nefedova Y, Cheng P, Alsina M, Dalton WS and Gabrilovich DI: Involvement of Notch-1 signaling in bone marrow stroma-mediated de novo drug resistance of myeloma and other malignant lymphoid cell lines. Blood 103: 3503-3510, 2004.

7. Han JH, Choi SJ, Kurihara N, Koide M, Oba Y and Roodman GD: Macrophage inflammatory protein-1alpha is an osteoclastogenic factor in myeloma that is independent of receptor activator of nuclear factor kappaB ligand. Blood 97: 3349-3353, 2001.

8. Abe M, Hiura K, Wilde J, Moriyama K, Hashimoto T, Ozaki S, Wakatsuki S, Kosaka M, Kido S, Inoue D and Matsumoto T: Role for macrophage inflammatory protein (MIP)-1alpha and MIP-1beta in the development of osteolytic lesions in multiple myeloma. Blood 100: 2195-2202, 2002.

9. Bergfeld SA and DeClerck YA: Bone marrow-derived mesenchymal stem cells and the tumor microenvironment. Cancer Metastasis Rev 29: 249-261, 2010.

10. Kassen D, Moore S, Percy L, Herledan G, Bounds D, Rodriguez-Justo M, Croucher P and Yong K: The bone marrow stromal compartment in multiple myeloma patients retains capability for osteogenic differentiation in vitro: Defining the stromal defect in myeloma. Br J Haematol 167: 194-206, 2014.

11. Noll JE, Williams SA, Tong CM, Wang H, Quach JM, Purton LE, Pilkington K, To LB, Evdokiou A, Gronthos S and Zannettino AC: Myeloma plasma cells alter the bone marrow microenvironment by stimulating the proliferation of mesenchymal stromal cells. Haematologica 99: 163-171, 2014.

12. Berenstein R, Blau O, Nogai A, Waechter M, Slonova E, Schmidt-Hieber M, Kunitz A, Pezzutto A, Doerken B and Blau IW: Multiple myeloma cells alter the senescence phenotype of bone marrow mesenchymal stromal cells under participation of the DLK1-DIO3 genomic region. BMC Cancer 15: 68, 2015.

13. Wallace SR, Oken MM, Lunetta KL, Panoskaltsis-Mortari A and Masellis AM: Abnormalities of bone marrow mesenchymal cells in multiple myeloma patients. Cancer 91: 1219-1230, 2001.
14. Corre J, Labat E, Espagnolle N, Hébraud B, Avet-Loiseau H, Roussel M, Huynh A, Gadelorge M, Cordelier P, Klein B, et al: Bioactivity and prognostic significance of growth differentiation factor GDF15 secreted by bone marrow mesenchymal stem cells in multiple myeloma. Cancer Res 72: 1395-1406, 2012.

15. Arnulf B, Lecourt S, Soulier J, Ternaux B, Lacassagne MN Crinquette A, Dessoly J, Sciaini AK, Benbunan M, Chomienne $\mathrm{C}$, et al: Phenotypic and functional characterization of bone marrow mesenchymal stem cells derived from patients with multiple myeloma. Leukemia 21: 158-163, 2007.

16. Li B, Shi M, Li J, Zhang H, Chen B, Chen L, Gao W, Giuliani N and Zhao RC: Elevated tumor necrosis factor-alpha suppresses TAZ expression and impairs osteogenic potential of Flk- $1^{+}$ mesenchymal stem cells in patients with multiple myeloma. Stem Cells Dev 16: 921-930, 2007.

17. André T, Najar M, Stamatopoulos B, Pieters K, Pradier O, Bron D, Meuleman N and Lagneaux L: Immune impairments in multiple myeloma bone marrow mesenchymal stromal cells. Cancer Immunol Immunother 64: 213-224, 2015.

18. Roccaro AM, Sacco A, Maiso P, Azab AK, Tai YT, Reagan M, Azab F, Flores LM, Campigotto F, Weller E, et al: BM mesenchymal stromal cell-derived exosomes facilitate multiple myeloma progression. J Clin Invest 123: 1542-1555, 2013.

19. Xu S, Cecilia Santini G, De Veirman K, Vande Broek I, Leleu X, De Becker A, Van Camp B, Vanderkerken K and Van Riet I: Upregulation of miR-135b is involved in the impaired osteogenic differentiation of mesenchymal stem cells derived from multiple myeloma patients. PLoS One 8: e79752, 2013.

20. Garayoa M, Garcia JL, Santamaria C, Garcia-Gomez A, Blanco JF, Pandiella A, Hernández JM, Sanchez-Guijo FM, del Cañizo MC, Gutiérrez NC and San Miguel JF: Mesenchymal stem cells from multiple myeloma patients display distinct genomic profile as compared with those from normal donors. Leukemia 23: 1515-1527, 2009.

21. Blackburn EH: Structure and function of telomeres. Nature 350: 569-573, 1991.

22. Harley $\mathrm{CB}$, Futcher $\mathrm{AB}$ and Greider $\mathrm{CW}$ : Telomeres shorten during ageing of human fibroblasts. Nature 345: 458-460, 1990.

23. Allsopp RC, Vaziri H, Patterson C, Goldstein S, Younglai EV, Futcher $\mathrm{AB}$, Greider $\mathrm{CW}$ and Harley $\mathrm{CB}$ : Telomere length predicts replicative capacity of human fibroblasts. Proc Natl Acad Sci USA 89: 10114-10118, 1992.

24. Zimmermann S, Voss M, Kaiser S, Kapp U, Waller CF and Martens UM: Lack of telomerase activity in human mesenchymal stem cells. Leukemia 17: 1146-1149, 2003.

25. Choumerianou DM, Martimianaki G, Stiakaki E, Kalmanti L, Kalmanti $\mathrm{M}$ and Dimitriou $\mathrm{H}$ : Comparative study of stemness characteristics of mesenchymal cells from bone marrow of children and adults. Cytotherapy 12: 881-887, 2010.

26. Banfi A, Bianchi G, Notaro R, Luzzatto L, Cancedda R and Quarto R: Replicative aging and gene expression in long-term cultures of human bone marrow stromal cells. Tissue Eng 8: 901-910, 2002

27. Ju Z, Jiang H, Jaworski M, Rathinam C, Gompf A, Klein C, Trumpp A and Rudolph KL: Telomere dysfunction induces environmental alterations limiting hematopoietic stem cell function and engraftment. Nat Med 13: 742-747, 2007.

28. Cawthon RM: Telomere measurement by quantitative PCR Nucleic Acids Res 30: e47, 2002.

29. Ci X, Li B, Ma X, Kong F, Zheng C, Björkholm M, Jia J and Xu D: Bortezomib-mediated down-regulation of telomerase and disruption of telomere homeostasis contributes to apoptosis of malignant cells. Oncotarget 6: 38079-38092, 2015.

30. Livak KJ and Schmittgen TD: Analysis of relative gene expression data using real-time quantitative PCR and the 2(-Delta Delta C(T)) method. Methods 25: 402-408, 2001.

31. Meyerson M, Counter CM, Eaton EN, Ellisen LW, Steiner P, Caddle SD, Ziaugra L, Beijersbergen RL, Davidoff MJ, Liu Q, et al: hEST2, the putative human telomerase catalytic subunit gene, is up-regulated in tumor cells and during immortalization. Cell 90: 785-795, 1997.

32. Reagan MR and Ghobrial IM: Multiple myeloma mesenchymal stem cells: Characterization, origin, and tumor-promoting effects. Clin Cancer Res 18: 342-349, 2012.

33. Akiyama M, Hideshima T, Hayashi T, Tai YT, Mitsiades CS, Mitsiades N, Chauhan D, Richardson P, Munshi NC and Anderson KC: Cytokines modulate telomerase activity in a human multiple myeloma cell line. Cancer Res 62: 3876-3882, 2002 . 
34. Bolzoni M, Donofrio G, Storti P, Guasco D, Toscani D, Lazzaretti M, Bonomini S, Agnelli L, Capocefalo A, Dalla Palma B, et al: Myeloma cells inhibit non-canonical wnt co-receptor ror2 expression in human bone marrow osteoprogenitor cells: Effect of wnt5a/ror2 pathway activation on the osteogenic differentiation impairment induced by myeloma cells. Leukemia 27: 451-463, 2013.

35. Li B, Fu J, Chen $\mathrm{P}$ and Zhuang $\mathrm{W}$ : Impairment in immunomodulatory function of mesenchymal stem cells from multiple myeloma patients. Arch Med Res 41: 623-633, 2010.

36. Lentzsch S, Gries M, Janz M, Bargou R, Dörken B and Mapara MY: Macrophage inflammatory protein 1-alpha (MIP-1 alpha ) triggers migration and signaling cascades mediating survival and proliferation in multiple myeloma (MM) cells. Blood 101: 3568-3573, 2003.
37. Vallet S, Pozzi S, Patel K, Vaghela N, Fulciniti MT, Veiby P, Hideshima T, Santo L, Cirstea D, Scadden DT, et al: A novel role for CCL3 (MIP-1 $\alpha$ ) in myeloma-induced bone disease via osteocalcin downregulation and inhibition of osteoblast function. Leukemia 25: 1174-1181, 2011.

38. Van Ziffle JA, Baerlocher GM and Lansdorp PM: Telomere length in subpopulations of human hematopoietic cells. Stem Cells 21: 654-660, 2003.

39. Samsonraj RM, Raghunath M, Hui JH, Ling L, Nurcombe V and Cool SM: Telomere length analysis of human mesenchymal stem cells by quantitative PCR. Gene 519: 348-355, 2013.

40. Ge W, Jiang J, Arp J, Liu W, Garcia B and Wang H: Regulatory T-cell generation and kidney allograft tolerance induced by mesenchymal stem cells associated with indoleamine 2,3-dioxygenase expression. Transplantation 90: 1312-1320, 2010. 